\title{
PROGRAMAS DE PREPARAÇÃO PARA APOSENTADORIA: UM ESTUDO NA FORD MOTOR COMPANY BRASIL
}

\author{
Retirement preparation programs: a study at Ford Motor Company Brazil
}

Programmes de préparation à la retraite: une étude chez Ford Motor Company au Brésil

Programas de preparación para la jubilación: un estudio en Ford Motor Company Brasil

\begin{abstract}
Izabel Cristina Rocha ${ }^{1}$
http://lattes.cnpq.br/6016456747225597.

Mestra em administração com ênfase em finanças pelas FECAP. Pós-graduada em controladoria e finanças pela Universidade Federal Fluminense (UFF) e em administração da produção e gestão da produtividade pela UNIARA. Graduada em Economia pela UNIARA. Fundação Escola de Comércio Álvares Penteado, São Paulo, Brasil
\end{abstract}

Ivam Ricardo Peleias ${ }^{2}$ http://lattes.cnpq.br/1583907056732686.

Doutor e Mestre em Ciências Contábeis pela Universidade de São Paulo-FEA-USP. Professor e pesquisador contábil do Centro Universitário, Fundação Es cola de Comércio Álvares Penteado (FECAP) e da Pontifícia Universidade Católica de São Paulo - PUC - SP. Fundação Escola de Comércio Álvares Penteado; Pontifícia Universidade Católica de São Paulo, São Paulo, São Paulo, Brasil.

\section{Cristineide Leandro Franca ${ }^{3}$ http://lattes.cnpq.br/2679994616132232.} Doutora e Mestra em Psicologia Clínica e Cultura. Psicóloga da Universidade de Brasília-UnB, Coordenadora de Atenção à Saúde e Qualidade de Vida-UnB. Pesquisadora do Grupo de Estudos em Prevenção e Promoção de Saúde no Ciclo de Vida, GEPPS Vida, Instituto de Psicologia, UnB. Universidade de Brasília, Brasília, Distrito Federal, Brasil.

Joels on Oliveira Sampaio ${ }^{4}$ http://lattes.cnpq.br/9134156549907160. Pós-doutorado USP. Doutor em Economia - USP. Doutor em Administração - FGV. Professor de finanças FECAP e FGV. Universidade Federal de São Paulo (UNIFESP), São Paulo, São Paulo, Brasil.

Jésus de Lisboa Gomes ${ }^{5}$ http://lattes.cnpq.br/3528255078888728. Doutor em Ciências Sociais (PUC/SP). Mestre em Administração de Empresas (FECAP). Professor e pesquisador do Mestrado profis sional em adminis tração do Centro Universitário - FECAP. Fundação Escola de Comércio Álvares Penteado, São Paulo, São Paulo, Brasil.

1 izarocha_bel@hotmail.com

2 ivamrp@fecap.br

3 cristineide@unb.br

4 joelsonsp@gmail.com

5 jgomes@fecap.br 


\title{
Resumo
}

Esta pesquisa analisou se um programa de preparação para aposentadoria promoveu clareza de objetivos e mudança no comportamento de planejamento para a aposentadoria, em 137 empregados, da Ford Motor Brasil, sendo 69 participantes do programa e 68 que não participaram. Duas escalas foram respondidas online:perspectiva de tempo futuro relativa à aposentadoria e mudança em comportamento de planejamento para aposentadoria. Realizou-se análise fatorial exploratória, teste de medidas independentes de dois grupos e regressão linear múltipla. $\mathrm{O}$ primeiro instrumento revelou uma estrutura unifatorial e o segundo quatro fatores. Não houve diferença significativa na comparação entre participantes e não-participantes do programa na mudança em comportamento de planejamento para aposentadoria. Constatou-se diferença significativa na clareza de objetivos para aposentadoria nos participantes do programa.

Palavras-chave: Preparação para aposentadoria; Perspectiva do tempo futuro; Envelhecimento; longevidade; Mudança de comportamento.

\begin{abstract}
Abs tract
The aim of this research was to analyze if a retirement preparation program clarified objetives and promoted a change in the behaviour planning for retirement in 137 employess of Ford Motor Brazil, in which 69 subjects participated and 68 did not participate. For this, two scales were answered online: future time perspective concerning retirement and a change in the behavior planning for retirement. It was made an exploratory factoring analysis, as well independent measures test of two groups, together with multiple linear regression. The first instrument revealed a unifactorial structure, and the second, four factors. There was no significant difference when comparing participants and non-participants of the behaviour planning for retirement program. It was stated a significant difference in the clarity of objectives for the participants retirement in the program.
\end{abstract}

Key-words: Retirement preparation; Future time perspective; Ageing; longevity; Behavior change.

\section{Resumé}

Cette étude a examiné si un programme de préparation à la retraite permettait de clarifier les objectifs et de modifier le comportement en matière de planification de la retraite de 137 employés de Ford Motor Brazil, avec 69 participants au programme et 68 non-participants. Les réponses en ligne ont été données à deux échelles: perspective du temps futur relatif à la retraite et changement de comportement de la planification à la retraite. Une analyse factorielle exploratoire, un test de mesures indépendantes de deux groupes et une régression linéaire multiple ont été effectués. Le premier instrument a révélé une structure unifamiliale et les deux autres facteurs. Il n'y avait pas de différence significative dans la comparaison entre les participants et les non-participants au programme dans le changement de comportement de la planification à la retraite. Il y avait une différence significative dans la clarté des objectifs de retraite des participants au programme.

Mots-clés: préparation à la retraite; Perspective du temps futur; Le vieillissement; la longévité; Changement de comportement.

\section{Resumen}

La finalidad de esta investigación fue analizar si un proyecto de preparación para jubilación ha promovido clareza de objetivos y cámbio en la conducta de planificación para jubilación de 137 empleados de Ford Motor Brasil, considerando que 69 han participado y 68 no lo han hecho. Dos niveles fueron contestados online: la perspectiva del tiempo futuro conectada a la jubilación, y el cámbio de conducta de planificación para jubilación. En esta investigación, ha sido hecha análisis de factores múltiple. La primera herramienta ha divulgado una estructura unifactorial, y la segunda, cuatro factores. No ha tenido grandes distinciones en la comparación entre los participantes y los que no han participado en el proyecto de cámbio de conducta de planificación para jubilación. Se ha contestado una gran distinción en la clareza de los objetivos para jubilación en los que han participado del proyecto.

Palabras-clave: Preparación para jubilación; Perspectiva del tiempo futuro; Envejecimiento; longevidad; Cámbio de conducta. 


\section{INTRODUÇÃO}

A população mundial vem envelhecendo e a expectativa de vida aumentando com o passar do tempo. Em 2017, a população global com 60 anos ou mais superava 962 milhões, mais de duas vezes superior à de 1980, quando era de 382 milhões. Espera-se que essa população idosa dobre mais uma vez até 1950, aproximando-se dos 2,1 bilhões. Em 2030, o número de idosos deverá superar o de crianças com menos de 10 anos (1,41 bilhão versus 1,35 bilhão). Em 2050 haverá mais pessoas com mais de 60 anos do que adolescentes com idade entre 10 e 24 anos (United Nations, 2017). No cenário nacional, em 2010, havia cerca de 19,6 milhões de idosos, devendo alcançar 66,5 milhões em 2050 (Simões, 2016).

No Brasil, a preocupação relativa às incertezas com a velhice contribuiu para a implantação do primeiro programa de acesso aos benefícios de previdência social promulgado pela Constituição Federal de 1824. No entanto, nos dias atuais, a previdência social é uma preocupação comum aos governantes e aos cidadãos das nações, pois é preciso equalizar as receitas, para reduzir as pressões sobre o orçamento público.

Organizações internacionais recomendam que, para uma política de envelhecimento saudável, o planejamento para a aposentadoria deve privilegiar a perspectiva da participação social, mobilidade e independência. Ademais, as políticas de recursos humanos devem considerar a permanência dos trabalhadores mais velhos, que desejam continuar na empresa após a idade regulamentar da aposentadoria (Organização Mundial de Saúde, 2015). Indicamse ainda ações que permitam ao empregado prestes a se aposentar fazer uma transição paulatina entre suas fases laboral e pós-aposentadoria (Organização Internacional do Trabalho, 2012) e, principalmente, a implantação do Programa de Preparação para Aposentadoria - PPA, para os que desejam se aposentar (Netto \& Netto, 2009).

No âmbito nacional, leis como a Política Nacional do Idoso (Lei 8.842/1994) e o Estatuto de Idoso (Lei 10.741/2003) recomendam a implantação de ações de preparação para aposentadoria nas organizações públicas e privadas. No serviço público, a Política de Atenção à Saúde e Segurança no Trabalho do Servidor Público Federal - PASS (MPOG, Portaria Normativa SRH $n^{\circ} 1.261 / 2010$ e $n^{\circ}$ 12/2018) também institui diretrizes para a promoção da educação para aposentadoria do servidor público. 


\section{Programas de Preparação para a Aposentadoria}

No cenário internacional, os programas de preparação para aposentadoria iniciaram nos Estados Unidos em meados de 1950, com as primeiras publicações na literatura ocorrendo nos anos de 1970. No Brasil, o PPA surge ao final dos anos de 1980, por iniciativa da Petrobrás, que na ocasião, começou a oferecer preparação para aposentadoria aos seus empregados (Leandro-França, Murta, Hershey, \& Barbosa, 2016). Portanto, em 1990, começam a ser produzidos estudos sobre programas de aposentadoria na literatura brasileira (França, 1992; Muniz, 1996; Zanelli, 1994).

Os programas de preparação para aposentadoria brasileiros costumam ter três formatos: continuado ou longo (duração de oito a 20 encontros, com periodicidade semanal, quinzenal ou mensal; intensivo (em regime de imersão, concentrado em dias consecutivos) e breve (duração de um a três encontros, uma vez por semana). Essas modalidades adotam técnicas educativas (ex. palestras, cartilhas) e vivencias (ex. uso de técnicas escritas, vídeos, música, depoimentos de pessoas aposentadas) e são realizadas, em geral, com grupos de pessoas que demonstram interesse comum em se preparar para essa período da vida (Seidl, Leandro-França, \& Murta, 2014). Os resultados desses programas mostram-se promissores em auxiliar os trabalhadores na transição para aposentadoria uma vez que promovem adaptação à aposentadoria, geram reflexões e conhecimento, assim como promovem mudanças positivas em saúde, qualidade de vida, atitudes e hábitos (França, 1992; Leandro-França et al, 2016; Zanelli, 1994).

\section{Modelos Teóricos na Preparação para Aposentadoria}

Os programas de preparação para aposentadoria são fundamentados em teorias como o Modelo Transteórico de Mudança (MTM), de Prochaska e DiClemente (1982), utilizado inicialmente, no Brasil, por França (2012), em estudos sobre desenvolvimento e avaliação de uma intervenção breve na preparação para a aposentadoria. Quanto ao Modelo de Perspectiva de Tempo Futuro (PTF), de Zimbardo e Boyd (1999), foi aplicado no Brasil em pesquisas sobre preparação para aposentadoria por Leandro-França, Iglesias e Murta (2018) para examinar evidências de validade de um instrumento que investiga a perspectiva de tempo futuro relativa à aposentadoria.

O modelo PTF exemplifica que há pessoas que definem objetivos-alvo a serem atingidos em um futuro distante e, por isso, possuem uma PTF extensa ou alongada, cujas características pessoais podem ser descritas de tal modo que: a) Para as pessoas com PTF extensa ou alongada, 
as distâncias temporais são percebidas de forma mais curta do que para as que possuem uma PTF restrita; b) As pessoas com PTF extensa ou alongada antecipam melhor as consequências em longo prazo de suas ações no presente, atribuem maior valor aos objetivos distantes e continuam motivadas para perseguir os objetivos; c) A satisfação, a perseverança e o esforço aplicados para executar uma tarefa são maiores nas pessoas com PTF extensa ou alongada. A probabilidade de passar da fase de plano para a ação também é maior nestas pessoas; d) As pessoas com PTF extensa ou alongada têm mais habilidades para converter seus desejos e vontades em ações comportamentais e posteriormente em ações (Lens, 1993).

$\mathrm{Na}$ associação desse modelo à preparação ou planejamento para aposentadoria, o entendimento sobre a perspectiva do tempo futuro é importante para a construção e elaboração do PPA. De acordo com a PTF, as pessoas com objetivos mais claros de futuro vivenciam maiores níveis de satisfação pessoal. Metas claras oferecem oportunidades de ampliar a intenção futura e orientam o comportamento desejado (Leandro-França, Murta, \& Iglesias, 2018).

O Modelo Transteórico de Mudança (MTM) busca analisar como as pessoas mudam seu comportamento ao longo do tempo, progredindo em direção à continuidade do comportamento saudável. Estes autores identificaram que os estágios de mudança (précontemplação, contemplação, preparação, ação, manutenção e término) envolvem processos de mudanças como: a tomada de consciência, a reavaliação de si mesmo, o engajamento em relação de ajuda, o gerenciamento de recompensas e esforços, entre outras (Reis \& Nakata, 2010).

Segundo Leandro-França, van Solinge, Hénkens, \& Murta (2016), as fases de mudança dos indivíduos no planejamento para aposentadoria se dividem em: 1) Pré-contemplação: não consideram possibilidades de mudança de comportamento e não tem consciência do que a mudança pode trazer para a sua vida; 2) Contemplação: estão conscientes da necessidade de mudar seus comportamentos, que a mudança é relevante, mas ainda não tomam iniciativa; 3) Preparação: pretendem tomar iniciativa para agir, e, às vezes implementam pequenas mudanças, que não são muito duradouras; 4) Ação: ocorrência real de mudança, em que a pessoa toma as decisões e se esforça de forma ativa para praticar novos comportamentos e atitudes; 5) Manutenção: quando a mudança de comportamento ocorre há pelo menos seis meses e a pessoa se esforça ao longo do tempo para consolidar os objetivos alcançados; 6) Término ou finalização: quando segue realizando o comportamento adquirido, por no mínimo cinco anos, tornando-o frequente e estável. Vale destacar que retrocessos ou recaídas podem 
ocorrer e são considerados típicos no processo de mudança de comportamento (Leandro-França et al., 2016).

\section{Hipóteses}

Na língua portuguesa, o termo "aposentar" provém da palavra hospedagem ou abrigo nos aposentos e remete à noção de ficar no interior da habitação. São sinônimos: reformar-se ou jubilar-se, o primeiro usado na carreira militar e o segundo no sentido de encher-se de júbilo, alegria; porém, o que parece ocorrer de fato é a negação do júbilo e da alegria conferida à ausência de trabalho, em função do significado expresso pela palavra reforma e pela noção de recolhimento a que o vocábulo aposento (aposentadoria) remete (Carlos, Jaques, Larratéa, \& Heredia, 1999). Assim, para muitas pessoas, este momento pode ser de sofrimento, insegura nça e por vezes surge o desejo de acabar com a própria vida ao chegar à fase da aposentadoria (Minayo, Pinto, Assis, Cavalcante, \& Mangas, 2012). Uma questão que se coloca é por que a aposentadoria deixa de ser um ato de júbilo, natural, para se tornar depreciativo?

A resposta a essa pergunta pode estar nas diferenças culturais. Nas culturas orientais, as pessoas idosas representam sabedoria e experiência. No ocidente, são estigmatizadas e rotuladas negativamente, de modo depreciativo, em contraponto ao valor dado aos jovens, tidos como ativos e produtivos. A perda do status de economicamente ativa pode gerar sofrimento e sensação de inadequação social, pois, para algumas pessoas, o trabalho representa poder, reconhecimento, status e produtividade. Desse modo, a aposentadoria pode gerar sentimentos de inferioridade, baixa autoestima, culpa e exclusão (Pereira \& Guedes, 2012).

Neste cenário, as empresas públicas e privadas têm um papel relevante, pois podem oferecer aos empregados educação e planejamento, tornando a aposentadoria um júbilo. $\mathrm{O}$ planejamento da aposentadoria torna-se mais eficaz quando os empregados podem participar dos Programas de Preparação para Aposentadoria (PPA) com antecedência (França, 2002). A organização pode instruir seus empregados sobre os aspectos relevantes da vida, desde a contratação até a aposentadoria, estimulando uma cultura organizacional mais consistente, contribuindo para o empregado realizar seu planejamento e preparando-o para se adaptar às mudanças que virão com a aposentadoria.

Este cenário, identificado em uma montadora de veículos, permitiu formular e buscar a seguinte resposta às questões de pesquisa deste estudo, assim decompostas: (1) Há diferenças entre os dois grupos de empregados da Ford Motor Company do Brasil quanto à participação em programas de preparação para aposentadoria no que se refere ao planejamento para 
aposentadoria? (2) Como a participação no PPA promoveu a conscientização sobre a importância do planejamento financeiro para a aposentadoria nos empregados da empresa?

Para responder às questões de pesquisa, foram formuladas as seguintes hipóteses:

- H1: Os participantes do PPA mudaram seu comportamento no planejamento para a aposentadoria, em comparação com os que não participaram do PPA.

- H2: Os participantes do PPA demonstraram melhor clareza de objetivos em relação ao tempo futuro, em comparação com os que não participaram do PPA.

- H3: Os participantes do PPA realizam mais contribuições adicionais ao plano de previdência privada, em comparação com os que não participaram do PPA.

A relevância social da pesquisa justifica-se, pois é preciso ampliar para a sociedade em geral ações que promovam uma aposentadoria bem-sucedida e estimular a discussão sobre educação financeira para o momento da aposentadoria. Este é um tema do qual "muitas pessoas se esquivam, como se fosse algo externo e que só diz respeito ao outro" (Pereira \& Guedes, 2012, p.161). Quanto à relevância científica, o presente estudo contribuirá para o avanço de pesquisas sobre preparação para aposentadoria, que tem aumentado nos últimos anos, mas, prevalece a necessidade de diversificar as abordagens e os métodos de pesquisa (Boehs, Medina, Bardagi, Luna, \& Silva, 2017).

Nesse contexto, o objetivo principal deste estudo é verificar como a participação em um PPA promoveu clareza de objetivos relativa à perspectiva de tempo futuro e mudança no comportamento de planejamento para a aposentadoria, em relação às questões financeiras, de saúde, qualidade de vida e aspecto psicossocial dos empregados da Ford Motor Company. O objetivo secundário é investigar como a participação em um PPA causa mudança de comportamento para que os empregados façam contribuições adịcionais no plano de previdência privada oferecido pela empresa.

\section{Método}

\section{Programa de PPA da Ford Motor Company Brasil}

A pesquisa foi feita com os empregados da Ford Motor Company, uma multinacional norte americana, com sede no estado americano de Michigan. No Brasil, há cinco unidades fabris: Camaçari/BA, Horizonte/CE, São Bernardo do Campo/SP, Tatuí/SP, Taubaté/SP, com 
mais de 10.000 empregados. Em 2015, a direção da Ford Motor Company aprovou a implementação de dois PPAs: Novos Rumos, com duração de cinco meses e com dois encontros mensais de quatro horas cada, no qual o empregado pode participar independentemente da idade e/ou tempo de serviço; e Tempo Certo, com duração de uma semana e com encontros diários de oito horas, dirigido a empregados com aposentadoria pelo INSS, que mantêm vínculo empregatício na Ford; e empregados aposentáveis, próximos da aposentadoria, por possuírem critérios de idade e tempo de contribuição para aposentar. Os programas são feitos em parceria com um fornecedor externo. Os temas abordados nos programas são semelhantes e distribuídos nos seguintes módulos: aspectos psicossociais da aposentadoria; saúde e qualidade de vida; aspectos médicos/orientação nutricional; gestão financeira à maturidade, legislação e opções pós-carreira/planejamento.

\section{Participantes}

A população foi formada pelos empregados das cinco unidades brasileiras. A amostra inicial pesquisada era de 350 empregados, sendo que 175 que participaram de um dos dois PPA, ao longo do biênio de 2015 e 2016; e outros 175 escolhidos por sorteio, que não participaram de nenhum dos programas. A amostra final foi de 137 respondentes à pesquisa, sendo que 69 que participaram de um dos programas e 68 que não participaram de nenhum PPA.

Foram identificadas e analisadas as características da amostra. Das respostas válidas obtidas, $39(28,46 \%)$ foram de mulheres e $98(71,5 \%)$ de homens. Foram identificadas cinco faixas etária: até 30 anos, com 16 sujeitos (11,68\%); de 31 a 40 anos, 56 (40,88\%), de 41 a 50 anos, 38 sujeitos $(27,74 \%)$ e de 51 a 60 anos, $25(18,24 \%)$ e acima de 60 anos, dois sujeitos $(1,46 \%)$. Quanto à faixa de renda, 34 sujeitos $(24,82 \%)$ recebiam entre $\mathrm{R} \$ 1.500,00$ e $\mathrm{R} \$ 5.000,00,67(48,90 \%)$ de $\mathrm{R} \$ 5.001,00$ a $\mathrm{R} \$ 10.000,00$, e $36(26,28 \%)$ entre $\mathrm{R} \$ 10.001,00$ e $\mathrm{R} \$ 20.000,00$. Não houve respondentes com renda superior a $\mathrm{R} \$ 20.000,00$. Em relação aos contribuintes para um plano de previdência privada, 96 respondentes $(70,07 \%)$ realizavam contribuição. A participação em um programa de preparação para a aposentadoria se mostrou equilibrada: 69 sujeitos $(50,36 \%)$ declaram participar, contra $68(49,4 \%)$ que informaram não participar. 


\section{Instrumentos}

Escala de Perspectiva de Tempo Futuro (EPTF): seu fundamento teórico é o modelo interdisciplinar de planejamento financeiro de Hershey, Jacobs-Lawson, McArdle e Hamagami (2007), Hershey, Henkens e Van Dalen (2010). O modelo possui um conjunto de fatores, psicológicos, econômico, financeiro, sócio demográfico e planejamento social, vistos como determinantes das práticas de planejamento para aposentadoria e preditores da percepção de poupar para o futuro. Os modelos de Hershey et al. (2007) e Hershey et al. (2010) possuem 4 dimensões: conhecimento de planejamento financeiro à aposentadoria, perspectiva do tempo futuro, clareza de ganho na aposentadoria e nível de atividade de planejamento em aposentadoria. No presente estudo, foram escolhidas as dimensões de conhecimento para planejamento financeiro de aposentadoria e clareza de ganho na aposentadoria, para avaliar se os empregados possuem clareza quanto aos objetivos à preparação para aposentadoria. $\mathrm{O}$ instrumento possui seis assertivas, com respostas de 1 a 5 (Escala Likert), variando de (1) discordo plenamente a (5) concordo plenamente, cujos itens escolhidos focam em reflexões, tais como: compreensão dos objetivos para aposentadoria, reflexão sobre o futuro e qualidade de vida na aposentadoria e propensão para definir e cumprir metas para o futuro.

\section{Escala de Mudança em Comportamento de Planejamento para Aposentadoria} (EMCPA): desenvolvido por Leandro-França et al. (2014), busca avaliar estágios de mudança (cognitivas, motivacionais e comportamentais), considerando comportamentos relevantes para uma aposentadoria bem-sucedida. Possui 15 assertivas, com categorias de respostas que variam de 1 a 6, pautadas nos estágios do Modelo Transteórico de Mudança: (1) não estou interessado nisso (pré-contemplação); (2) venho pensando em fazer algo sobre isso (contemplação); (3) estou decidido a fazer algo nesse sentido (preparação); (4) comecei a fazer, mas parei (recaída); (5) comecei a fazer há pouco tempo (ação); e (6) já faço isso há bastante tempo (manutenção).

A EMCPA possui dois fatores: 1) investimento ocupacional-social, tais como: participar de grupos na comunidade, investir em projetos que podem ser feitos a partir da aposentadoria; fazer trabalho voluntário na comunidade e 2) investimento em autonomia e bem-estar como: realizar atividade física regularmente, ter tempo de investir na vida familiar, praticar atividades de lazer, ter investimentos financeiros para o futuro. 


\section{Procedimentos de coleta e análise de dados}

Realizou-se uma análise comparativa com dois grupos de empregados da empresa: um que participou do PPA com outro que não participou do programa. A coleta de dados ocorreu com aplicação dos instrumentos por meio da plataforma Google Forms. Os 350 empregados receberam por e-mail, uma carta convite para participarem da pesquisa. Destes, 25 não possuíam e-mail; assim, receberam a carta convite e a pesquisa impressa para que pudessem participar, caso optassem. Os empregados tiveram duas semanas para responderem ao questionário. Para obter um maior número de participantes, o período foi estendido por mais cinco dias. O e-mail continha um Termo de Consentimento Livre e Esclarecido para adesão ao estudo, explicações sobre os objetivos, procedimentos da pesquisa e sobre o anonimato dos dados. Os servidores que concordaram em participar responderam ao instrumento indicado em link específico.

Para tratamento dos dados, utilizaram-se análises estatísticas descritivas e inferenciais, (SPSS na versão 22). Foram verificados os pressupostos das análises estatísticas univariadas e multivariadas, dados atípicos, dados ausentes, distribuição normal das variáveis e multicolinearidade. Após a análise dos pressupostos teóricos, verificaram-se as características psicométricas das escalas usadas por meio da análise fatorial exploratória. Foi realizada uma Regressão Múltipla Padrão (RMP) para verificar a relação de predição entre as variáveis socioprofissionais e os escores das escalas (Hair, Black, Babin, Anderson, \& Tatham, 2009). Para testar a hipótese de que há diferença entre os que participaram ou não do PPA, foi realizado o teste-t para medidas independentes, comparando os escores das escalas. Foi verificado se o número de pessoas que fazem contribuições adicionais à previdência privada é maior entre os que participaram do PPA, por meio do teste Qui². Em todas as análises, testes bicaudais com o valor de $\mathrm{P}<0,05$ foram considerados estatisticamente significativos.

\section{Resultados}

\section{Propriedade psicométrica das escalas}

O critério da distância de Mahalanobis revelou 15 casos extremados multivariados. Mesmo com a retirada dos extremados, a proporção entre o número de participantes e o de itens manteve-se adequada para testar a solução fatorial acima de 5 casos (Hair et al., 2009). Por isso, optou-se pela exclusão de tais casos, dando continuidade às analises e considerando-se 129 casos validados. 
O resultado do teste de Kaiser-Meyer-Olkin (KMO) confirmou a adequação da amostra para a escala EPTF, obtendo-se um valor ótimo (de =0,889). O KMO da EMCPA $(0,626)$ não retornou valores adequados. Mesmo com índices de KMO baixo, a solução fatorial se tornou adequada após ajuste no modelo fatorial, por meio das cargas fatoriais. Confirmou-se a presença de intercorrelações entre os itens pelo teste de esfericidade de Bartlett para as duas escalas, EPTF $(\chi 2=336,922 ; \mathrm{gl}=15 ; \mathrm{p}<0,001)$ e EMCPA $(\chi 2=428,52 ; \mathrm{gl}=105 ; \mathrm{p}<0,001)$, sendo ratificada pelo valor da determinante da Matriz, por ser diferente de zero, o que mostra não se tratar de uma matriz identidade.

Para a escala EPTF, o critério do autovalor identificou uma estrutura unifatorial. Apenas o primeiro componente apresentou variância que superasse a variância de um item isolado $(\gamma=3,484)$. Na escala EMCPA, pelo critério, o autovalor foi identificado até 5 fatores. Para confirmar a quantidade de fatores a serem extraídos, realizou-se a análise paralela pela simulação Monte-Carlo. Comparando-se os autovalores dos dados reais com os aleatórios constatou-se que a escala EPTF possui uma estrutura unifatorial e que a escala EMCPA teria a retenção de 04 fatores, os quais possuíam dados reais com autovalores maiores do que dos dados aleatórios. A extração dos fatores da escala EPTF foi feita sem a necessidade de rotação, pois se trata de uma estrutura unifatorial. Optou-se por suprimir itens com carga fatorial menor que $\pm 0,30$. Assim, o item 06, "Economizar dinheiro para o futuro foi uma importante lição que aprendi quando criança" não permaneceu na solução fatorial.

Pode-se notar, na Tabela 1, que foram mantidos cinco itens na solução fatorial. $O$ conteúdo dos cinco itens da escala de EPTF $(\gamma=3,095$; Variância Explicada VE $=51,578 \%$; $\alpha$ $=0,876$ ) remete a clareza dos objetivos na preparação para aposentadoria. As cargas dos itens variaram entre 0,617 e 0,895 . Tabela 1: Estrutura Fatorial da Escala de Percepção de Tempo Futuro

4 Estou economizando o suficiente para me aposentar
confortavelmente

$5 \mathrm{Eu}$ fiz cálculos para estimar quanto dinheiro preciso economizar para me aposentar confortavelmente

3 Eu tenho uma visão clara de como será minha vida na aposentadoria.

1 Eu tenho estabelecido metas de quanto economizar para a 0,739 aposentadoria

2 Eu sei mais do que a maioria das pessoas sobre planejamento $\quad 0,617 \quad 0,499$ para a aposentadoria 
Variância Explicada $51,578 \quad 51,578$

Alpha Cronbach $(\alpha) \quad 0,876$

Nota. $\mathrm{h}^{2}-$ Comunalidade.

Para a EMCPA houve a extração dos quatro fatores, usando a fatoração dos componentes principais com rotação oblíqua, método oblimin, por ser de uma análise exploratória (Hair et al., 2009). Após a rotação para a extração dos fatores da escala de EMCPA, o item 12 "Fazer cursos de aperfeiçoamento em minha área" apresentou carga fatorial inferior a 0,30 , sendo retirado da solução fatorial. Foi estipulado que itens presentes em mais de um fator permanecessem onde tivessem maior carga. Como apresentado na Tabela 2, foram mantidos na solução fatorial 14 itens. $\mathrm{O}$ fator $01(\gamma=1,90$; Variância Explicada $-\mathrm{VE}=16,99 \%$; $\alpha=0,652$ ) agrupou quatro itens que remetem à intenção de dedicação ao grupo familiar, com cargas entre 0,466 e 0,630 . O fator $02(\gamma=1,566 ; \mathrm{VE}=11,329 \% ; \alpha=0,565)$ agrupou 04 itens que remetem à intenção de dedicação ao convívio com a comunidade, com cargas entre 0,338 e 0,625 . O fator $03(\gamma=1,414 ; \mathrm{VE}=6,223 \% ; \alpha=0,703)$ agrupou dois itens que remetem à intenção de dedicação as novas áreas profissionais, com cargas entre 0,617 e 0,856. O fator 04 $(\gamma=1,920 ; \mathrm{VE}=5,794 \% ; \alpha=0,684)$ agrupou 04 itens que remetem à intenção de dedicação à saúde, com cargas entre 0,412 e 0,743 . A variância total explicada pela estrutura dos 4 fatores da escala de EMCPA foi de 40,344\%.

Tabela 2: Estrutura fatorial da escala de mudança em comportamento de planejamento para aposentadoria

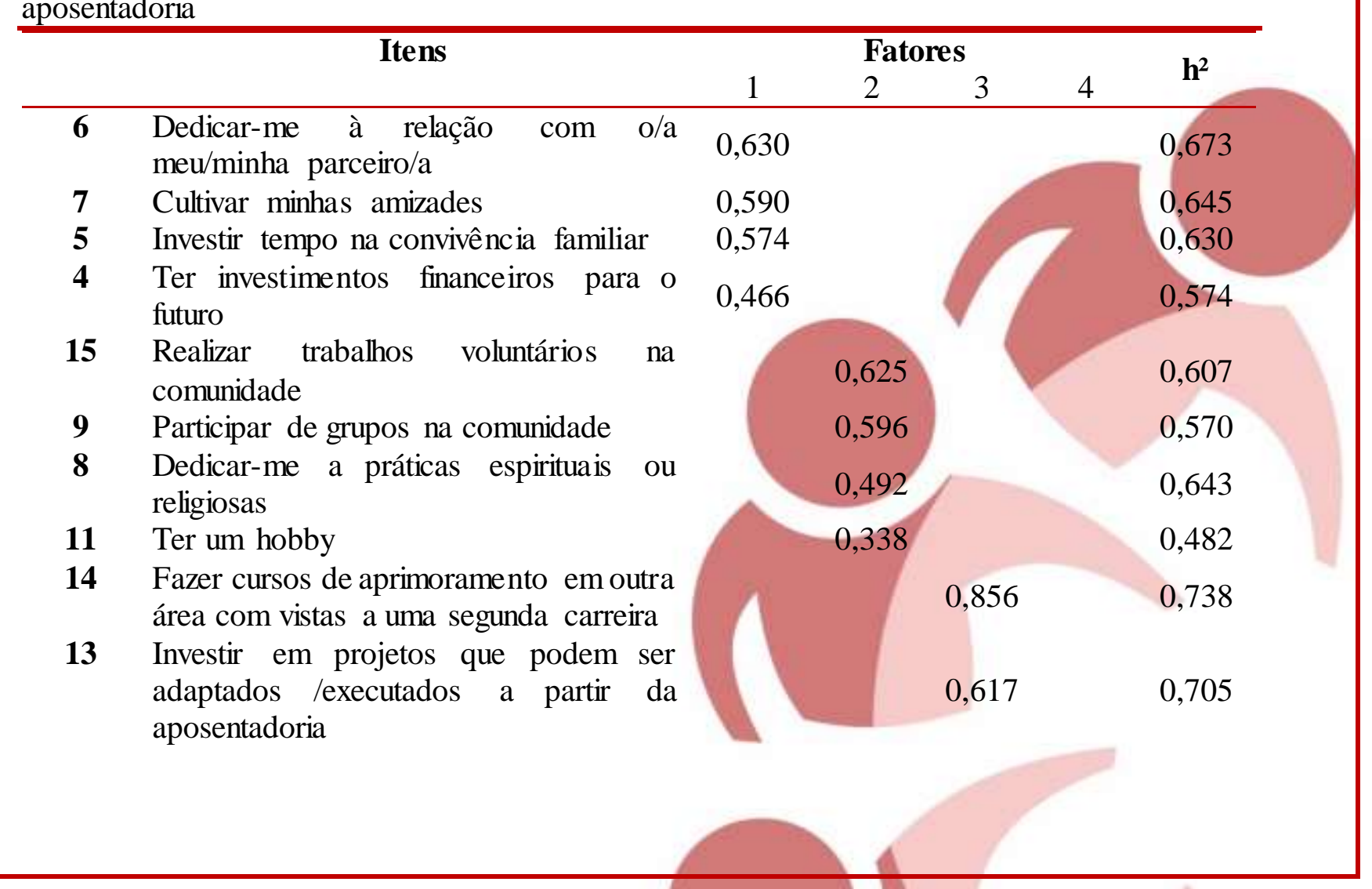


1 Praticar atividade física regularmente

$$
0.743 \quad 0,688
$$

2 Ter uma alimentação mais saudável

$$
\begin{array}{cc}
- & 0,638
\end{array}
$$

10 Praticar atividades de lazer

3 Fazer consultas e exames médicos de check-up

$\begin{array}{rccccc} & & & & 0,412 & 0,626 \\ \text { Número de itens } & 04 & 04 & 02 & 04 & 14 \\ \text { Autovalor } & 1,900 & 1,566 & 1,414 & 1,920 & - \\ \text { Variância Explicada } & 16,998 & 11,329 & 6,223 & 5,794 & 40,344 \\ \text { Alpha Cronbach } & 0,652 & 0,565 & 0,703 & 0,684 & -\end{array}$

Nota. $\mathrm{h}^{2}-$ Comunalidade.

A Tabela 3 descreve os escores médios, obtidos pelo método de média, para cada fator. Nessa análise foram inseridos todos os indivíduos da amostra. Em geral, a amostra se mostrou homogênea em relação aos escores das escalas. Apenas o fator Dedicação a novas áreas da escala EMCPA $\left(\mathrm{C}_{v}=0,388\right)$ teve coeficiente de variância $\geq 0,30$. Entre os fatores da escala EMCPA, o fator Dedicação ao grupo familiar teve a maior média (média $=5,39 \pm 0,62 ; \mathrm{n}=$ 137). O fator Dedicação a novas áreas teve a menor média (média $=3,21 \pm 1,25 ; \mathrm{n}=137$ ). As correlações entre os fatores da Escala EMCPA foram de magnitude pequena, o que demonstra que a análise fatorial foi capaz de fazer distinção da variância entre os fatores. O escore da escala EPTF teve média de 3,12 ( $\pm 0,89 ; \mathrm{n}=137)$.

Tabela 3: Média e correlação de Pearson das variáveis de estudo ( $\mathrm{n}=137)$.

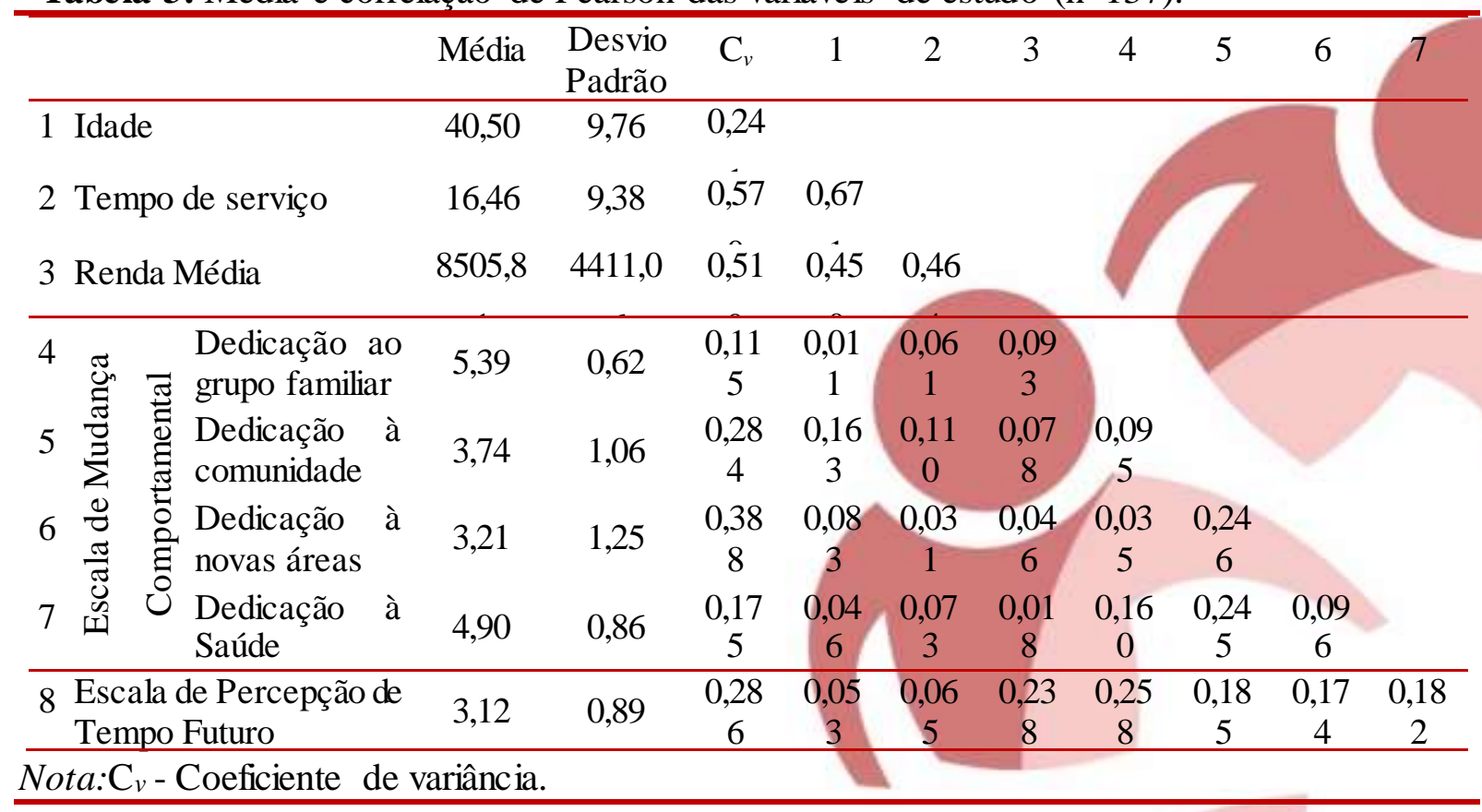


Ao comparar a população que participou do PPA e a que não participou, constatou-se diferença significativa apenas na escala de EPTF $\left(t_{(127)}=2,657 ; p=0,009\right)$, sendo que a que participou (média $=3,31 \pm 0,79 ; \mathrm{n}=68$ ) teve média maior em relação àquela que não participou (média $=2,90 \pm 0,95 ; \mathrm{n}=61)$. Nenhum fator da escala EMCPA teve diferença significativa $(\mathrm{p}$ $>0,05)$.

O teste Qui² busca avaliar quantitativamente a relação entre os resultados. Na comparação com os que fazem contribuições adicionais para a previdência privada, o teste de Qui² não identificou diferenças significativas entre os que participaram do PPA e os que não participaram $(\chi 2=1,275 ; \mathrm{gl}=1 ; \mathrm{p}=0,259)$.

Foram identificados casos com dados extremados univariados $(n=8)$ e utilizado o critério da distância de Mahalanobis, multivariados ( $\mathrm{n}=6)$. Por esse motivo, 14 casos foram excluídos da amostra e restando123 válidos. Tal exclusão não prejudicou a continuidade das análises, pois o tamanho da amostra mostrou-se adequado para obter tamanhos de efeito médios $( \pm 0,30)$ com nível de significância $(\alpha)$ de 0,01 ou 0,05 .

O escore da escala EPTF e o fator Dedicação a novas áreas da escala EMCPA foram os únicos que apresentara aderência à curva normal. Como a normalidade na variável dependente é um pressuposto que não deve ser violado na regressão múltipla, optou-se por realizar as regressões apenas para essas variáveis (Hair et al., 2009).

Não se identificou altos níveis de colinearidade entre as variáveis previsoras. Analisando-se tal pressuposto, verificou-se: ausência de correlações acima de 0,90 na matriz de correlações; fator de inflação da variância (FIV) de cada variável abaixo de cinco; estatística tolerância de cada variável acima de 0,20.

O modelo 01 (Predição da escala de percepção de tempo futuro) é responsável por $11,2 \%$ da variação no escore da escala EPTF. A diferença entre o valor de $\mathrm{R}^{2}$ e $\mathrm{R}^{2}$ ajustado mostra que na população geral provavelmente esse modelo explique 3,5\% menos de variância. O tempo de serviço e a renda média foram as variáveis do modelo que se mostraram preditoras significativas $(p<0,05)$. A mudança na variância forneceu uma razão $F$ maior do que um e significativa $\left(\mathrm{F}_{(5,118)}=11,783 ; \mathrm{p}<0,01\right)$, mostrando que a predição não se deve ao acaso. Ver Tabela 4. 
Tabela 4: Modelo de predição do escore da escala EPTF a partir das variáveis sócioprofissionais $(\mathrm{n}=123)$.

\begin{tabular}{cccc}
\hline & \multicolumn{3}{c}{ Percepção de Tempo Futuro } \\
\cline { 2 - 4 } & \multicolumn{3}{c}{ Modelo 01 } \\
\cline { 2 - 4 } & Beta & $\mathrm{T}$ & Sig. \\
\hline Idade & 0,104 & 0,884 & 0,378 \\
Tempo de serviço & $-0,289$ & $-2,406$ & 0,018 \\
Renda Média & 0,337 & 3,516 & 0,001 \\
Sexo & 0,071 & 0,802 & 0,424 \\
Estado Civil & $-0,103$ & $-1,165$ & 0,246 \\
\hline $\mathrm{R}$ & & 0,335 & \\
$\mathrm{R}^{2}$ & & 0,112 & \\
$\mathrm{R}^{2}$ ajustado & & 0,077 &
\end{tabular}

Nota. R - Coeficiente de regressão; $\mathrm{R}^{2}$ - Coeficiente de determinação; B - Gradiente de Inclinação; t - Teste t-student; Sig. - Grau de significância; Sexo - 1 feminino, 2 Masculino; Estado Civil - 0 solteiro, 1 Casado. Método Entrada Forçada.

A análise dos resíduos demonstrou bom ajuste do modelo, indicando que pode ser generalizado para outras populações. Foi identificado independência dos termos de erro, homocedasticidade da variância e linearidade do fenômeno. Como também que não existia autocorrelação serial entre eles (Durbin-Watson = 2,097). Os resíduos apresentaram distribuição normal multivariada, de acordo com o teste Kolmogorov-Smirnov (KS = 0,046; gl $=120 ; \mathrm{p}=0,200)$.

O modelo 02 (Predição do escore do fator dedicação à comunidade da escala EMCPA) é responsável por 3,3\% da variação no escore do Fator Dedicação à comunidade da escala EMCPA. A diferença entre o valor de $\mathrm{R}^{2}$ e $\mathrm{R}^{2}$ ajustado mostra que, na população geral, provavelmente esse modelo explique $2,9 \%$ menos de variância. Ver Tabela 5.

Tabela 5: Modelo de predição do escore do Fator Dedicação à comunidade da escala EMCPA, a partir das variáveis sócioprofissionais $(n=123)$

\begin{tabular}{lccc}
\hline & \multicolumn{3}{c}{ Dedicação à comunidade } \\
\cline { 2 - 4 } & \multicolumn{3}{c}{ Modelo 02 } \\
\cline { 2 - 4 } & Beta & T & Sig. \\
\hline Idade & 0,150 & 1,232 & 0,220 \\
Tempo de serviço & $-0,013$ & $-0,109$ & 0,914 \\
Renda Média & 0,018 & 0,176 & 0,860 \\
Sexo & 0,053 & 0,579 & 0,564
\end{tabular}




\begin{tabular}{|c|c|c|c|}
\hline Estado Civil & 0,029 & 0,320 & 0,749 \\
\hline $\mathrm{R}$ & & 0,182 & \\
\hline $\mathrm{R}^{2}$ & & 0,033 & \\
\hline $\mathrm{R}^{2}$ ajustado & & 0,004 & \\
\hline
\end{tabular}

Nota: R - Coeficiente de regressão; R2 - Coeficiente de determinação; B - Gradiente de Inclinação; $\mathrm{t}$ - Teste t-student; Sig. - Grau de significância; Sexo - 1 feminino, 2 Masculino; Estado Civil - 0 solteiro, 1 Casado; Método Entrada Forçada.

A mudança na variância forneceu uma razão $F \geq 1$ e significativa $\left(F_{(5,118)}=5,276\right.$; $\mathrm{p}=0,490$ ), mostrando que a predição pode ser devida ao acaso. Desse modo, não foi realizada análise dos resíduos, uma vez que o modelo 02 não se mostrou um bom preditor.

\section{Discussão}

O trabalho buscou verificar em que medida a participação de empregados da Ford Motor Company Brasil, em dois programas de preparação para aposentadoria, promoveu clareza de objetivos e mudança no comportamento de planejamento para a aposentadoria. A análise fatorial exploratória das escalas EPTF e EMCPA mostrou uma boa adequação e validação, corroborando com os resultados encontrados em Leandro-França et al. (2016, 2018). Os dados da escala de percepção do tempo futuro, que remete à clareza dos objetivos na preparação para aposentadoria, considerando o índice de consistência interna, são superiores aos obtidos em Hershey et al. (2007) e alguns itens do estudo de Leandro-França et al. (2018).

As altas cargas fatoriais dos itens: "Eu tenho estabelecido metas de quanto economizar para a aposentadoria $(0,739)$ ", "Eu fiz cálculos para estimar quanto dinheiro preciso economizar para me aposentar confortavelmente $(0,804)$ ", "Eu tenho uma visão clara de como será minha vida na aposentadoria 0,798)" e "Eu estou economizando o suficiente para me aposentar confortavelmente $(0,895)$ " apontam indícios que os empregados têm perspectiva futura extensa e clareza dos objetivos para a preparação da aposentadoria. Examinando o escore médio das escalas, em relação à percepção do tempo futuro, percebe-se que os empregados da Ford têm clareza em relação aos objetivos da aposentadoria.

Ao analisar os resultados das regressões no modelo EPTF, observa-se significância nas variáveis: tempo de serviço e renda média, indicando que, quanto maior o tempo de serviço, menor a clareza dos objetivos na preparação para aposentadoria. Inversamente contrário em relação à variável renda média, cujo resultado mostra que quanto maior a renda, maior é a clareza em relação aos objetivos na preparação para aposentadoria. 
Analisando a variável tempo médio de serviço, é possível verificar que as pessoas que trabalham há mais tempo na Ford Motor Company podem não ter clareza em relação aos objetivos da preparação para aposentadoria. Colaborando para isso o fato de estarem mais próximos dela e não terem se planejado, não terem planos para aposentadoria e tenderem a se acomodar. Assim, sugere-se que o programa de preparação para aposentadoria da Ford Motor Company possa ampliar a discussão sobre metas ou planos para aposentadoria especialmente para os que trabalham há mais tempo nessa organização.

Quanto à variável renda, os resultados apontam que quanto maior a remuneração, maior a clareza de objetivos em relação à aposentadoria. Uma hipótese para essa questão é que estes empregados de maior renda tenham mais clareza em relação às perdas financeiras ao se aposentar. Logo, por saberem que precisarão complementar a aposentadoria para manter o mesmo padrão de vida enquanto ativo, estima-se que esses empregados estão investindo nos seus objetivos para o pós-carreira.

Ainda com relação à variável clareza dos objetivos para a aposentadoria, quando comparados os grupos quem participou e não participou do PPA, a análise do Teste t para médias independentes indica diferença entre os grupos. Isto significa que os grupos que participaram do PPA têm mais clareza em relação aos objetivos da aposentadoria.

A estrutura de mudança de comportamento em planejamento para aposentadoria resultou em quatro fatores que remetem à: intenção de dedicação ao grupo familiar, dedicação ao convívio com a comunidade, dedicação às novas áreas profissionais e dedicação à saúde. Ao analisar a consistência interna, comparando os dados encontrados no estudo atual com os dados de Leandro-França et al. (2014), os resultados são equivalentes. Quanto à carga fatorial, o maior resultado para "Fazer cursos de aprimoramento em outra área com vistas a uma segunda carreira" $(0,856)$ evidencia que os empregados pretendem fazer alguma ação para ter uma segunda carreira; assim, podem ainda estar na fase de contemplação como demonstrado no Modelo Transteórico de Mudança (Leandro-França et al, 2016).

Considerando a escala de mudança em comportamento de planejamento para aposentadoria e os estágios de mudança (contemplação, preparação, ação, manutenção e recaída), os fatores dedicação às novas áreas profissionais e à comunidade evidenciam que os empregados estão decididos a fazer alguma mudança nesse sentido, demonstrando que estão na fase de preparação. No fator dedicação à saúde, há indícios de que os empregados iniciaram a ação de se cuidar, mas não deram continuidade ao comportamento adquirido, evidenciando uma recaída. O fator dedicação ao grupo familiar revela que os empregados iniciaram alguma mudança há pouco tempo, indicando que estão na fase da ação. Dados semelhantes foram 
obtidos no estudo de Leandro-França et al. (2014). Os resultados descritos corroboram com a literatura. Em seus estudos, Leandro-França et al. (2016) destacam que os comportamentos como retrocessos ou recaídas podem ocorrer e são considerados característicos do processo de mudança de comportamento.

Quando comparados os grupos quem participou e não participou dos Programas, a análise do Teste t para médias independentes comparou se, para cada uma das escalas, há diferença de média entre o grupo que participou e o que não participou do PPA. Os resultados indicam que para a escala de mudança em comportamento de planejamento não há diferenças significativas.

Considerados os objetivos e as hipóteses deste estudo, a partir das análises não paramétricas refutou-se H1, que afirmava que participantes do PPA realizaram mudanças de comportamento no planejamento para a aposentadoria, em comparação com os que não participaram do PPA. Por outro lado, não se rejeitou $\mathrm{H} 2$ que afirmava que os empregados participantes do PPA revelaram melhor clareza de objetivos quanto ao tempo futuro, em comparação com os que não participaram do PPA. Assim, a H2 foi confirmada. O objetivo secundário foi verificar em que medida a participação em um PPA promove mudança de comportamento para que os empregados realizem contribuições adicionais no plano de previdência privada oferecido pela empresa. Com base nas análises não paramétricas, refutouse H3, a qual afirma que os empregados participantes do PPA realizam mais contribuições adicionais ao plano de previdência privada, em comparação com os que não participaram do PPA.

Como limitações, o presente estudo não realizou o pré-teste com os participantes do PPA para que fosse possível analisar a percepção antes e depois da participação no Programa, como também, não fez a exploração da variável escolaridade. Desse modo, recomenda-se que pesquisas futuras contemplem essas lacunas.

Destacam-se, como principais contribuições deste estudo, a adoção de um método comparativo em uma amostra robusta, a utilização de escalas com evidências de validação na população brasileira e de estatística paramétrica (pouco usual em pesquisas da área). Compreende-se a relevância em reforçar a necessidade de realizar estudos futuros sobre o tema, incipiente no Brasil, principalmente em organizações privadas. Uma razão para a carência nas pesquisas é a dificuldade de acessar dados sobre ações direcionadas às organizações privadas (Panozzo \& Monteiro, 2013). O acesso a essas informações é essencial para ampliar o escopo de pesquisas nesse tipo de organização e para incentivar a implantação de programas de PPA, vislumbrando aumentar o conhecimento e a compreensão dos empregados sobre o tema. 


\section{Considerações Finais}

A partir do questionamento inicial "Há diferenças entre dois grupos de empregados da Ford Motor Company do Brasil, participantes e não participantes de um PPA, oferecido pela organização, no que se refere à clareza de objetivos relativa à perspectiva de tempo futuro e mudança no comportamento de planejamento para a aposentadoria?" Constatou-se, a não ocorrência de mudança nos participantes dos dois grupos quanto ao comportamento de planejamento para aposentadoria. Por outro lado, houve mudança significativa quanto à clareza em relação aos objetivos na preparação para aposentadoria para os empregados que participaram do PPA.

Para pesquisas futuras, sugere-se replicar este estudo, após dois ou três anos, utilizando os mesmos instrumentos, com a realização de entrevistas e método misto de análise dos dados para obter uma visão ampla e integrada sobre as ações que os empregados estão realizando em relação à preparação para aposentadoria. Com o uso de entrevistas e análise qualitativa dos dados será possível verificar em qual estágio de mudança o empregado encontra-se na escala de mudança em comportamento de planejamento para aposentadoria como realizado em estudo de Leandro-França et al. (2016).

Por fim, sugere-se que novas abordagens, como a Employee Value Proposition - EVP (em português, Proposição de Valor do Empregado), sejam utilizadas como aporte teórico em estudos sobre evidências de validade de instrumentos com foco no planejamento para aposentadoria. O EVP é uma abordagem alinhada às estratégias de planejamento da força de trabalho. A proposição de valor deve identificar as políticas, processos e programas que revelem o compromisso da empresa com o crescimento dos empregados, desenvolvimento de gestão e reconhecimento contínuo dos serviços. O EVP descreve como a empresa apoia o empregado e sua família com benefícios, programas, oportunidades de aprendizagem e de carreira, a cultura da empresa para empregados atuais e potenciais (Minchington, 2011). Essa abordagem demonstra, com atributos, o que é oferecido aos empregados para estimular que se desenvolvam e sejam reconhecidos. A preparação para aposentadoria, viabilizada por meio de programas oferecidos nas organizações, pode ser considerada um atributo do EVP por se tratar de um benefício oferecido pela empresa ao empregado. Tendo a preparação para a aposentadoria como atributo do EVP, as empresas conseguem melhorar seu plano de sucessão, pois a aposentadoria deixaria de ser um tabu, para ser algo natural e que precisa ser planejado pelos empregados e pelas empresas. 


\section{Referências}

Boehs, S., Medina, P., Bardagi, M., Luna, I., \& Silva, N. (2017). Revisão da literatura latinoamericana sobre aposentadoria e trabalho: perspectivas psicológicas. Revista Psicologia: Organizações e Trabalho, 17(1), 54-61.

Carlos, S. A., Jaques, M. G., Larratéa, S. V., \& Heredia, O. C.(1999). Identidade, aposentadoria e terceira idade. Estudos Interdisciplinares sobre o envelhecimento, 1, 77-87.

França, C. L. (2012). Modelo de uma intervenção breve para planejamento da aposentadoria: Desenvolvimento e avaliação (Dissertação de Mestrado). Universidade de Brasília, Brasilia, DF, Brasil.

França, L. H. (1992). Terceira idade: O trabalho social com idosos no SESC e os programas de preparação para aposentadoria nas empresas. Revista de Administração Pública, 26, 174181.

França, L. H. (2002). Repensando a aposentadoria com qualidade: Um manual para facilitadores em programas de educação para a aposentadoria. Rio de Janeiro: CRDE/UnATI/UER.

França, L. H., Nalin, C., Siqueira-Brito, A., Amorim, S., Rangel, T., \& Ekman, N. C. (2014). A percepção dos gestores brasileiros sobre os programas de preparação para aposentadoria. Estudos interdisciplinares sobre o envelhecimento, 19(3), 879- 898.

Hair, J. F., Black, B., Babin, B., Anderson, R. E., \&Tatham, R. L. (2009). Multivariate data Analysis. Upper Saddle River: Prentice Hall.

Hershey, D. A., Jacobs-Lawson, J. M., McArdle, J. J., \& Hamagami, A. (2007). Psychologic al foundations of financial planning for retirement. Journal of Adult Development, 14(1-2), 26-36.

Hershey, D. A., Henkens, K., \& Van Dalen, H. (2010). Aging and financial planning for retirement: Interdisciplinary influences viewed through a cross-cultural lens. International Journal of Aging and Human Development, 70(1), 1-38. doi: 10.2190/AG.70.1.a.

Leandro-França, C., Iglesias, F., \& Murta, S. G. (2018). Futuro e aposentadoria: evidências de validade para uma medida de perspectiva temporal. Revista Psicologia: Organizações e Trabalho, 18(2), 1-6.

Leandro-França, C., Murta, S., Hershey, D., \& Barbosa, L. (2016). Evaluation of retirement planning programs: A qualitative analysis of methodologies and efficacy. Educational Gerontology, 42(7), 497-512.

Leandro-França, C., van Solinge, H., Henkens, K., \& Murta, S. (2016). Effects of three types of retirement preparation program: A qualitative study of civil servants in Brazil. Educational Gerontology, 42, 388-400. doi:10.1080/03601277.2016.1139969.

Leandro-França, C., Murta, S., \& Iglesias, F. (2014). Planejamento da aposentadoria: Uma escala de mudança de comportamento. Revista Brasileira de Orientação Profissional, 15(1), 75-84. 
Lei 8.842/1994, de 4 de janeiro de 1994. Dispõe sobre a política nacional do idoso, cria o Conselho Nacional do Idoso e dá outras providências. Recuperado de: http://www.planalto.gov.br/ccivil_03/leis/L8842.htm.

Lei 10.741/2033, de 1 de outubro de 2003. Dispõe sobre o Estatuto do Idoso e dá outras providências. Recuperado de: http://www.planalto.gov.br/ccivil 03/leis/2003/L10.741.htm.

Lens, W. (1993). La signification motivationnelle de la perspective future. Revuequébécoise de psychologie, 14(1), 69-83.

Minchington, B. (2011). Employer branding without borders: A pathway to corporate success. Journal of Corporate Recruiting Leadership, 7(6), 3-14.

Muniz, J. A. (1996). PPA: Programa de preparação para o amanhã. Estudos de Psicologia, 2(1) 198-204.

Mynayo, M. C. S, Pinto, L. W, Assis, S. G, Cavalcante, F. G., \& Mangas, R. M. N. (2012). Tendência da mortalidade por suicídio na população brasileira e idosa, 1980-2006, Revista de Saúde Pública, 46(2), 300-309.

Organização Internacional do Trabalho - OIT (2012). As boas práticas brasileiras em seguridade social (Vol. 1). Brasilia: OIT.

Organização Mundial da Saúde - OMS (2015). Resumo do Relatório Mundial de Envelhecimento e Saúde. Brasîlia, DF: Organização Mundial da Saúde.

Panozzo, E. A. L., \& Monteiro, J. K. (2013). Aposentadoria e saúde mental: Uma revisão de literatura. Cadernos de Psicologia Social do Trabalho, 16(2), 199-209.

Pereira, T. M. F. R. A., \& Guedes, S. S. (2012). Novo Tempo: A Experiência de Implantação do Programa de Preparação para o Pós-Carreira no IFRN. Revista Holos,04(28), 158-177.

Portaria $n^{\circ} 1.261$ de 05 de maio de 2010. Institui os Princípios, Diretrizes e Ações em Saúde Mental que visam orientar os órgãos e entidades do Sistema de Pessoal Civil - SIPEC da Administração Pública Federal sobre a saúde mental dos servidores. Recuperado de: http://www.trtsp.jus.br/gera1/tribunal2/ORGAOS/Min_Div/MPOG_Port1261_10.html.

Portaria Normativa $n^{\circ} 12$, de 20 de outubro de 2018. Institui as diretrizes gerais de promoção da educação para aposentadoria do servidor público federal, que visam orientar os órgãos e entidades do Sistema de Pessoal Civil da Administração Federal - SIPEC. Recuperado de: $\quad$ http://planejamento.gov.br/noticias/planejamento-publica-diretrizes-de-sobreeducacao-para-a-aposentadoria.

Prochaska, J., \& Diclemente, C. (1982). Transtheoretical therapy: Toward a more integrative model of change. Psychotherapy: Theory, Research \& Practice, 19(3), 276-288. doi.org/10.1037/h0088437.

Reis, G. G., \& Nakata, L. E. (2010). Modelo Transteórico de Mudança: Contribuições para o coaching de Executivos. Revista Brasileira de Orientação Profissional, 11(01), 61-72. 
Seidl, J., Leandro-França, C., \& Murta, S. (2014). Formatos de programas de educação para aposentadoria. In S. Murta, C. Leandro-França, \& J. Seidl (Eds.). Programas de educação para aposentadoria: como planejar, implementar e avaliar (pp. 84-113). Novo Hamburgo: Sinopsys.

Simões, C. C. S. (2016). Relações entre as alterações históricas na dinâmica demográfica brasileira e os impactos decorrentes do processo de envelhecimento da população (Estudos $e$ análises: Informação demográfica e socioeconômica, 4). Rio de Janeiro: IBGE, Coordenação de População e Indicadores Sociais.

United Nations Population Division (2013). World References Population Ageing 2013.New York: United Nations Population Division.

Zimbardo, P. G., \& Boyd, J. N. (1999). Putting time in perspective: A valid, reliable individualdifferences metric. Journal of Personality and Social Psychology, 77,1271-1288. doi: 10.1037/0022-3514.77.6.1271.

Zanelli, J. C. (1994). Aposentadoria: Percepções dos servidores da Universidade Federal de Santa Catarina. Anais do EnANPAD - Universidade de São Paulo, São Paulo, SP. 